\title{
Controle de Helicoverpa armigera (Lepidoptera: Noctuidae) em soja com inseticidas químicos e biológicos
}

\author{
Cassiano Carlos Kuss(1), Rejane Cristina Roppa Kuss Roggia(2), Claudir José Basso(1), \\ Maria Cristina Neves de Oliveira ${ }^{(3)}$, Osmar Henrique de Castro Pias ${ }^{(1)}$ e Samuel Roggia ${ }^{(3)}$
}

\begin{abstract}
(1)Universidade Federal de Santa Maria, Centro de Educação Superior Norte do Rio Grande do Sul, Departamento de Ciências Agronômicas e Ambientais, Linha Sete de Setembro, s/no, BR 386, Km 40, Caixa Postal 54, CEP 98400-000 Frederico Westphalen, RS, Brasil. E-mail: cassianok2012@gmail.com, claudirbasso@gmail.com, henriquepias@yahoo.com.br ${ }^{(2)}$ Autônoma, Avenida José Gabriel de Oliveira, no 915, Torre 1, Apartamento 304, CEP 86047-360 Londrina, PR, Brasil. E-mail: rkuss2003@yahoo.com.br ${ }^{(3)}$ Embrapa Soja, Rodovia Carlos João Strass, s/no, Acesso Orlando Amaral, Distrito de Warta, Caixa Postal 231, CEP 86001-970 Londrina, PR, Brasil. E-mail: mcristina.oliveira@embrapa.br, samuel.roggia@embrapa.br
\end{abstract}

Resumo - O objetivo deste trabalho foi avaliar o desempenho de inseticidas autorizados emergencialmente para o controle de Helicoverpa armigera (Lepidoptera: Noctuidae) em soja. Sete inseticidas foram pulverizados em campo e, após 24 horas, folhas do ponteiro foram coletadas e oferecidas para lagartas de $2 \mathrm{o}$ instar em laboratório. Lagartas do $4^{\circ}$ instar receberam a última folha trifoliolada que se encontrava completamente expandida no momento da pulverização. Outro grupo foi exposto a folhas coletadas a partir de 72 horas da pulverização. Em campo, seis inseticidas foram pulverizados e, em seguida, as plantas foram infestadas com lagartas de $2^{\circ}$ e 30 instar. No primeiro estudo, flubendiamida, clorantraniliprole, clorfenapir, indoxacarbe e metoxifenozida causaram $100 \%$ de mortalidade do $4^{\circ}$ instar aos oito dias após o início da exposição, enquanto baculovírus e Bacillus thuringiensis (Bt) propiciaram mortalidade de 60-75\%, que evoluiu para $88-90 \%$ ao final da fase de pupa. Para o 20 instar, apenas flubendiamida e clorantraniliprole proporcionaram mortalidade de $100 \%$. Flubendiamida, clorantraniliprole e clorfenapir apresentaram o menor tempo letal para o $4^{\circ}$ instar, e flubendiamida e clorantraniliprole, para o 2o instar. Após 72 horas da pulverização, o desempenho dos inseticidas foi insatisfatório. Em campo, houve

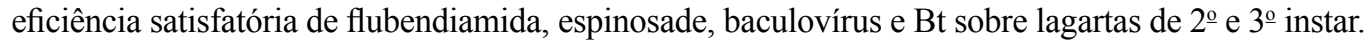

Termos para indexação: Glycine max, ameaça fitossanitária, Heliothinae, manejo integrado de pragas, praga invasora, tempo letal.

\section{Control of Helicoverpa armigera (Lepidoptera: Noctuidae) in soybean with chemical and biological insecticides}

\begin{abstract}
The objective of this work was to evaluate the performance of insecticides authorized on an emergency basis to control of Helicoverpa armigera (Lepidoptera: Noctuidae) in soybean. Seven insecticides were sprayed on the field and, 24 hours after that, soybean pointer leaves were collected and offered to $2^{\text {nd }}$ instar larvae in the laboratory. Fourth instar larvae received the last trifoliate leaf that was fully expanded at the time of spraying. Another larvae group was exposed to leaves collected from 72 hours onwards after spraying. In the field, six insecticides were sprayed, and then the plants were infested with $2^{\text {nd }}$ and $3^{\text {rd }}$ instar larvae. In the first study, flubendiamide, chlorantraniliprole, chlorfenapyr, indoxacarb, and methoxyfenozide caused $100 \%$ mortality of the $4^{\text {th }}$ instar, eight days after the beginning of exposure, while baculovirus and Bacillus thuringiensis (Bt) caused $60-75 \%$ mortality, which reached $88-90 \%$ at the end of the pupal stage. For $2^{\text {nd }}$ instar larvae, only flubendiamide and chlorantraniliprole caused $100 \%$ mortality. Flubendiamide, chlorantraniliprole, and chlorfenapyr showed the lowest lethal time for the $4^{\text {th }}$ instar, and flubendiamide and chlorantraniliprole for the $2^{\text {nd }}$ instar. Seventy-two hours after spraying, the performance of insecticides was not satisfactory. In the field, there was satisfactory efficiency of flubendiamide, spinosad, baculovirus, and $\mathrm{Bt}$ on $2^{\text {nd }}$ and $3^{\text {rd }}$ instar larvae.
\end{abstract}

Index terms: Glycine max, phytosanitary threat, Heliothinae, integrated pest management, invasive pest, lethal time.

\section{Introdução}

Helicoverpa armigera (Hübner) (Lepidoptera: Noctuidae) é uma espécie com ampla distribuição geográfica na Europa, Ásia, África e Oceania (European and Mediterranean Plant Protection Organization, 2006).
Em 2013 foi relatada pela primeira vez nas Américas, no Brasil, nos estados de Goiás, Mato Grosso, Bahia e Paraná (Czepak et al., 2013; Specht et al., 2013; Tay et al., 2013); antes disso, era considerada praga quarentenária. Poucos meses depois foi registrada no Paraguai (Senave..., 2013) e Argentina (Murúa et al., 2014). 
Cunningham \& Zalucki (2014) listaram 177 gêneros de plantas hospedeiras de $H$. armigera, entre as quais há várias espécies de importância econômica para o Brasil, como soja (Glycine max), algodão (Gossypium hirsutum), milho (Zea mays), trigo (Triticum aestivum), arroz (Oryza sativa), aveia (Avena sativa), sorgo (Sorghum bicolor), feijão (Phaseolus vulgaris), amendoim (Arachis hypogaea), girassol (Helianthus annuus), tomate (Lycopersicon esculentum), batata (Solanum tuberosum) e guandu (Cajanus cajan).

As lagartas de $H$. armigera atacam tanto estruturas vegetativas quanto reprodutivas de seus hospedeiros, como cotilédones, folhas, flores e frutos (Johnson \& Zalucki, 2005). Essa espécie apresenta ampla capacidade de dispersão (Farrow \& Daly, 1987), pode migrar para uma distância de até $1.000 \mathrm{~km}$ (Pedgley, 1985), consegue sobreviver em condições adversas (Karim, 2000; Mironidis et al., 2010) e tem alto potencial reprodutivo (Naseri et al., 2009a, 2009b). Tais características, associadas à ampla distribuição geográfica e diversidade de plantas hospedeiras, possibilitam elevada capacidade de disseminação e têm contribuído para sua rápida adaptação às diversas regiões agrícolas brasileiras, oferecendo riscos para diferentes sistemas produtivos.

A lagarta $H$. armigera é considerada uma das principais pragas agrícolas no mundo, e estimamse prejuízos anuais de US\$ 5 bilhões em virtude de seu ataque (Lammers \& MacLeod, 2007). Diante da incidência generalizada de $H$. armigera no Brasil e do risco de perdas causadas pelo seu ataque, o Ministério da Agricultura, Pecuária e Abastecimento (Mapa) publicou, até meados de novembro de 2014, pelo menos 22 atos normativos relacionados à declaração de estado de emergência fitossanitária, permissão de importação, uso emergencial e registro especial temporário (RET) de inseticidas, visando à supressão da praga e minimização dos seus danos em culturas agrícolas no País (Sislegis, 2014). O Mapa concedeu a permissão de uso emergencial de inseticidas químicos e biológicos de diversos grupos químicos e mecanismos de ação para controle de Helicoverpa spp. Na cultura da soja, antes de 2013, praticamente não existiam inseticidas registrados para Helicoverpa spp., então a definição dos inseticidas e doses a serem autorizados foi baseada no que se conhece para o controle da praga em outros países ou na lista de inseticidas registrados no Brasil para outras espécies de lagartas em soja e em outras culturas. Diante da importância de $H$. armigera para a soja, são necessários estudos mais detalhados do desempenho desses inseticidas nessa cultura.

O objetivo deste trabalho foi avaliar o desempenho de inseticidas autorizados emergencialmente para o controle de Helicoverpa armigera (Lepidoptera: Noctuidae) em soja.

\section{Material e Métodos}

O experimento foi conduzido na Embrapa Soja, em Londrina, PR $\left(23^{\circ} 11^{\prime} \mathrm{S}, 51^{\circ} 11^{\prime} \mathrm{W}\right)$, em uma área de 0,35 ha semeada com a cultivar de soja BMX Turbo RR, em 17/2/2014.

Os tratamentos consistiram em diferentes inseticidas, aplicados no limite inferior da dose registrada no Mapa para o controle de Helicoverpa spp. em soja:


esporos por hectare de Bacillus thuringiensis, var. kurstaki, linhagem HD-1, Dipel WP, (Valent BioSciences Corporation, Osage, IA, EUA), inseticida biológico; T3, 4X1011 corpos de oclusão por hectare do vírus da poliedrose nuclear HzSNPV (Gemstar, Certis USA, Columbia, MD, EUA), inseticida biológico; T4, 10,00 $\mathrm{g} \mathrm{ha}^{-1}$ de clorantraniliprole, Premio, (DuPont do Brasil S.A., Barueri, SP), do grupo químico diamida; T5, 24,00 $\mathrm{g} \mathrm{ha}^{-1}$ de flubendiamida, Belt, (Bayer Brasil, São Paulo, SP), do grupo químico diamida; T6, 21,60 $\mathrm{g} \mathrm{ha}^{-1}$ de metoxifenozida, Intrepid $240 \mathrm{SC}$, (Dow Agrosciences Industrial Ltda., São Paulo, SP), do grupo químico diacilhidrazina; T7, 192,00 $\mathrm{g} \mathrm{ha}^{-1}$ de clorfenapir, Pirate, (Basf S.A., São Paulo, SP), do grupo químico análogo de pirazol; T8, 60,00 $\mathrm{g} \mathrm{ha}^{-1} \mathrm{de}$ indoxacarbe, Avatar, (DuPont do Brasil S.A., Barueri, SP), do grupo químico oxadiazina.

A pulverização foi realizada em 14/3/2014 sobre plantas de soja no estádio vegetativo V3 (Herman, 1985), com volume de calda de $160 \mathrm{~L} \mathrm{ha}^{-1}$, pressão de 0,5 Mpa (50 Psi) e pontas TJ 110.015 , sob $29,2^{\circ} \mathrm{C}, 65 \%$ de umidade relativa do ar e vento de $7,5 \mathrm{~km} \mathrm{~h}^{-1}$. Foram utilizadas parcelas de $7 \times 20 \mathrm{~m}$, com margem mínima de $2 \mathrm{~m}$ entre parcelas para reduzir os riscos de deriva durante a pulverização dos tratamentos.

A partir das plantas tratadas em campo, foram colhidas as folhas do ponteiro ( $4^{\circ}$ nó da planta a partir da base) e oferecidas às lagartas de $H$. armigera de 2o instar em laboratório. A exposição das lagartas aos tratamentos iniciou 24 horas após a pulverização, e diariamente novas folhas do $4^{\circ}$ nó foram coletadas 
em campo e fornecidas para as lagartas até o final da fase larval. As lagartas foram obtidas de criação em laboratório e foram mantidas previamente sob dieta artificial, adaptadas de Greene et al. (1976), sem os anticontaminantes. O mesmo procedimento foi adotado para lagartas de 4o instar, porém, coletandose folhas do 3 o nó da planta, que correspondeu à última folha trifoliolada, que se encontrava no topo do dossel e completamente expandida no momento da pulverização.

Com o objetivo de avaliar o possível efeito residual dos tratamentos, outro grupo de lagartas foi exposto aos tratamentos a partir das 72 horas após a pulverização. Durante o período do experimento (14/3 a 8/4), as médias da temperatura e umidade do ar foram de $23,2^{\circ} \mathrm{C}$ e $81,7 \%$, a média da radiação global total foi de $15,7 \mathrm{MJ} \mathrm{m}^{-2}$, e a precipitação pluviométrica acumulada foi de $142 \mathrm{~mm}$. Houve precipitação pluviométrica aos 4, 5, 7, 8, 16, 17 e 18 dias após a aplicação dos produtos, com os seguintes volumes, respectivamente: $5,5,26,46,4,46$ e $10 \mathrm{~mm}$.

Em laboratório, foi utilizado o delineamento inteiramente casualizado, com 10 repetições para cada tratamento. Cada unidade experimental foi composta por uma lagarta individualizada em copo de PVC de $100 \mathrm{~mL}$ com tampa, contendo folhas de soja de cada tratamento. As lagartas foram mantidas a $27 \pm 2^{\circ} \mathrm{C}$ e sob fotoperíodo de $12 \mathrm{~h}: 12 \mathrm{~h}$ (luz:escuro). Ao longo do estudo foi avaliada a mortalidade diária de lagartas, mortalidade da fase de lagarta e de pupa, biomassa e deformações de pupas - esses dois últimos representam possíveis efeitos subletais proporcionados pelos tratamentos. As deformações de pupas foram avaliadas atribuindo-se notas em uma escala de zero a três para cada uma das deformações descritas por Rodrigues Filho (1985), sendo zero, um e dois as notas para deformação severa, intermediária e leve, respectivamente, e três ausência de deformação. Foram avaliados separadamente 14 tipos de deformações que foram agrupados em uma única nota média de deformação de pupas por repetição. Com base nos dados coletados, foi calculada a mortalidade acumulada aos 3 e 8 dias após o início da exposição das lagartas aos tratamentos, pela fórmula de Schneider-Orelli (Püntener, 1981). A biomassa e deformações de pupas foram analisadas estatisticamente pelo método não paramétrico de Kruskal-Wallis (Campos, 1979), e as médias dos tratamentos comparadas por DMS e teste de $\chi_{(\mathrm{gl} ; 0,05)}^{2}$. Foi calculado tempo letal para atingir $50 \%$ e $80 \%$ de mortalidade de lagartas (TL50 e TL80) pela análise de Logit, e para a comparação dos tratamentos foram considerados os intervalos de confiança (limites inferior e superior) obtidos ao nível de 5\% de significância.

Adicionalmente foi conduzido um experimento com infestação de lagartas de $H$. armigera de $2^{\circ}$ e $3^{\circ}$ instar em campo para avaliar a eficiência dos seguintes produtos e doses: $1,25 \times 10^{13}$ esporos ha ${ }^{-1}$ de Bacillus thuringiensis, var. kurstaki, linhagem HD-1, utilizandose as marcas comerciais Dipel WP e Dipel, (Valent BioSciences Corporation, Osage, IA, EUA), e Best HD (Laboratório Farroupilha, Patos de Minas, MG); $4 \times 10^{11}$ corpos de oclusão ha ${ }^{-1}$ do vírus da poliedrose nuclear HzSNPV (Gemstar, Certis USA, Columbia, MD, EUA); 24,00 $\mathrm{g} \mathrm{ha}^{-1}$ de espinosade, Tracer, (Dow AgroSciences Industrial Ltda., São Paulo, SP); e 24,00 $\mathrm{g} \mathrm{ha}^{-1}$ de flubendiamida, Belt, (Bayer Brasil, São Paulo). Neste foram utilizadas parcelas de $6 \times 20 \mathrm{~m}$, com margem mínima de $2 \mathrm{~m}$ entre parcelas para reduzir os riscos de deriva no momento da pulverização. Foi utilizado o delineamento de blocos ao acaso, e cada tratamento teve quatro repetições. Em cada parcela, 10 plantas de soja no estádio vegetativo V2 (Herman, 1985) foram infestadas com lagartas, e cada planta foi identificada com uma pequena estaca para facilitar sua localização posteriormente. Os produtos foram pulverizados entre as $16 \mathrm{e}$ 17 horas, nos dias 18 e 14/11/2014, sob 30,8 e $27,3^{\circ} \mathrm{C}, 36,2$ e $49,8 \%$ de umidade relativa do ar e vento de 10,5 e $13,8 \mathrm{~km} \mathrm{~h}^{-1}$, respectivamente, para lagartas de $2^{\circ}$ e $3^{\circ}$ instar. Para a pulverização, foi utilizada a mesma tecnologia de aplicação da primeira etapa.

Imediatamente após a secagem da calda de pulverização, cada planta com uma lagarta foi protegida por uma tela com objetivo de excluir o efeito de predação natural e possibilitar a avaliação do efeito do produto sobre a lagarta, com o mínimo de interferências externas. Aos 3 e 8 dias após a pulverização, foi realizada a avaliação dos tratamentos, e a mortalidade acumulada foi calculada pela fórmula de Schneider-Orelli (Püntener, 1981). Os valores de eficiência, em decimal, foram transformados por arco seno de $\mathrm{x}^{0,5}$. Em seguida, foram submetidos à análise de variância (Anova), e as médias foram comparadas pelo teste de Tukey a $5 \%$ de probabilidade. 


\section{Resultados e Discussão}

Para as lagartas de 4 instar que receberam folhas colhidas a partir de 24 horas da pulverização, a melhor eficiência de controle foi observada nos tratamentos compostos por flubendiamida, clorantraniliprole, clorfenapir e indoxacarbe com $100 \%$ de mortalidade acumulada aos 3 dias de exposição (Tabela 1). Essas observações indicam que existem produtos eficientes para o controle de $H$. armigera, com bom desempenho, inclusive para lagartas de $4 \div$ instar, que são consideradas de difícil controle. $\mathrm{O}$ desempenho satisfatório desses produtos foi observado também por Chatterjee \& Mondal (2012) e Abbas et al. (2015) para H. armigera.

Para o regulador de crescimento de insetos, metoxifenozida, foram necessários 8 dias para que a mortalidade atingisse $100 \%$. Isso indica que o inseticida também é eficiente no controle da praga, como também observado por Alavo et al. (2011), porém, é relativamente mais lento em virtude de seu mecanismo de ação que atua na metamorfose da lagarta (Dhadialla, 2012).

Tabela 1. Mortalidade acumulada, biomassa e deformação de pupa em virtude da exposição de lagartas de Helicoverpa armigera do $2^{\circ}$ e $4^{\circ}$ instar a inseticidas biológicos e químicos, a partir de 24 e 72 horas da pulverização destes em plantas de soja, em Londrina, PR, safra agrícola 2013/2014.

\begin{tabular}{|c|c|c|c|c|c|c|}
\hline \multirow[t]{2}{*}{ Tratamento } & \multicolumn{4}{|c|}{ Mortalidade acumulada $(\%)^{(1)}$} & \multicolumn{2}{|c|}{ Parâmetros de pupa ${ }^{(2)}$} \\
\hline & 3 dia & 8o dia & Fase larva & Fase de pupa & Biomassa $(\mathrm{g})$ & Deformação \\
\hline & \multicolumn{6}{|c|}{24 horas após a aplicação - lagarta de 4o instar } \\
\hline Testemunha & - & - & - & - & $0,24 \mathrm{a}$ & $2,95 \mathrm{a}$ \\
\hline Bacillus thuringiensis & 60,00 & 60,00 & 80,00 & 90,00 & $0,23 \mathrm{a}$ & - \\
\hline Baculovírus & 0,00 & 75,00 & 75,00 & 87,50 & $0,22 \mathrm{a}$ & $2,91 \mathrm{a}$ \\
\hline Clorantraniliprole & 100,00 & 100,00 & 100,00 & 100,00 & - & - \\
\hline Flubendiamida & 100,00 & 100,00 & 100,00 & 100,00 & - & - \\
\hline Metoxifenozida & 30,00 & 100,00 & 100,00 & 100,00 & - & - \\
\hline Clorfenapir & 100,00 & 100,00 & 100,00 & 100,00 & - & - \\
\hline \multirow[t]{2}{*}{ Indoxacarbe } & 100,00 & 100,00 & 100,00 & 100,00 & - & - \\
\hline & \multicolumn{6}{|c|}{24 horas após a aplicação - lagarta de $2^{\circ}$ instar } \\
\hline Testemunha & - & - & - & - & $0,30 \mathrm{a}$ & $3,00 \mathrm{a}$ \\
\hline Bacillus thuringiensis & 0,00 & 0,00 & 40,00 & 60,00 & $0,24 \mathrm{a}$ & $2,96 \mathrm{a}$ \\
\hline Baculovírus & 0,00 & 30,00 & 30,00 & 70,00 & $0,28 \mathrm{a}$ & $2,93 \mathrm{a}$ \\
\hline Clorantraniliprole & 100,00 & 100,00 & 100,00 & 100,00 & - & - \\
\hline Flubendiamida & 100,00 & 100,00 & 100,00 & 100,00 & - & - \\
\hline Metoxifenozida & 30,00 & 57,00 & 66,67 & 100,00 & $0,28 \mathrm{a}$ & $3,00 \mathrm{a}$ \\
\hline Clorfenapir & 40,00 & 40,00 & 60,00 & 70,00 & $0,25 \mathrm{a}$ & $2,88 \mathrm{a}$ \\
\hline \multirow[t]{2}{*}{ Indoxacarbe } & 0,00 & 10,00 & 80,00 & 80,00 & $0,28 \mathrm{a}$ & $2,98 \mathrm{a}$ \\
\hline & \multicolumn{6}{|c|}{72 horas após a aplicação - lagarta de $4^{\circ}$ instar } \\
\hline Testemunha & - & - & - & - & $0,25 \mathrm{a}$ & $2,91 \mathrm{a}$ \\
\hline Bacillus thuringiensis & 0,00 & 44,00 & 44,44 & 55,56 & $0,27 \mathrm{a}$ & $2,96 \mathrm{a}$ \\
\hline Baculovírus & 10,00 & 11,00 & 11,11 & 33,33 & $0,28 \mathrm{a}$ & $2,96 \mathrm{a}$ \\
\hline Clorantraniliprole & 10,00 & 40,00 & 70,00 & 80,00 & $0,28 \mathrm{a}$ & $2,96 \mathrm{a}$ \\
\hline Flubendiamida & 30,00 & 40,00 & 40,00 & 40,00 & $0,29 \mathrm{a}$ & $3,00 \mathrm{a}$ \\
\hline Metoxifenozida & 0,00 & 40,00 & 80,00 & 80,00 & $0,30 \mathrm{a}$ & $3,00 \mathrm{a}$ \\
\hline Clorfenapir & 20,00 & 40,00 & 66,67 & 66,67 & $0,23 \mathrm{a}$ & $2,96 \mathrm{a}$ \\
\hline \multirow[t]{2}{*}{ Indoxacarbe } & 0,00 & 0,00 & 40,00 & 50,00 & $0,27 \mathrm{a}$ & $2,99 \mathrm{a}$ \\
\hline & \multicolumn{6}{|c|}{72 horas após a aplicação - lagarta de $2^{\underline{0}}$ instar } \\
\hline Testemunha & - & - & - & - & $0,28 \mathrm{a}$ & $2,88 \mathrm{a}$ \\
\hline Bacillus thuringiensis & 0,00 & 0,00 & 0,00 & 10,00 & $0,28 \mathrm{a}$ & $2,98 \mathrm{a}$ \\
\hline Baculovírus & 0,00 & 10,00 & 20,00 & 50,00 & $0,30 \mathrm{a}$ & $2,80 \mathrm{a}$ \\
\hline Clorantraniliprole & 0,00 & 30,00 & 40,00 & 50,00 & $0,30 \mathrm{a}$ & $2,95 \mathrm{a}$ \\
\hline Flubendiamida & 0,00 & 30,00 & 30,00 & 60,00 & $0,29 \mathrm{a}$ & $2,99 \mathrm{a}$ \\
\hline Metoxifenozida & 10,00 & 10,00 & 20,00 & 30,00 & $0,30 \mathrm{a}$ & $2,97 \mathrm{a}$ \\
\hline Clorfenapir & 0,00 & 10,00 & 10,00 & 10,00 & $0,28 \mathrm{a}$ & $2,98 \mathrm{a}$ \\
\hline Indoxacarbe & 0,00 & 0,00 & 44,44 & 44,44 & $0,31 \mathrm{a}$ & $2,91 \mathrm{a}$ \\
\hline
\end{tabular}

${ }^{(1)}$ Mortalidade acumulada no 3o dia e no $8^{\circ}$ dia após o início da exposição aos tratamentos e ao final da fase de larva e de pupa, calculada pela fórmula de Schneider-Orelli (Püntener, 1981). ${ }^{(2)}$ Massa de pupa avaliada entre as 24 e 48 horas iniciais da fase e deformação de pupa avaliada com notas de 0 a 3 (Rodrigues Filho, 1985), em que o valor máximo corresponde à ausência de deformação, e, para ambos os parâmetros, as médias seguidas de letras iguais na coluna não diferem entre si pelo teste de Kruskal-Wallis, $\chi_{(7 ; 0,05)}^{2}$ a $5 \%$ de significância. 
No tratamento com Bacillus thuringiensis (Bt), a mortalidade acumulada atingiu $60 \%$ com 3 dias de exposição das lagartas de 4을 instar, sem aumentar com o passar do tempo, o que indica que a eficiência do Bt foi definida com poucos dias após a aplicação. Isso está relacionado ao seu mecanismo de ação, que agride o epitélio intestinal, interrompendo a alimentação da lagarta em poucas horas após o início da exposição, evoluindo para infecção generalizada e morte das lagartas em 2 a 4 dias (Bueno et al., 2012). É possível, também, que as chuvas ocorridas a partir do terceiro dia possam ter contribuído para a redução dos propágulos do patógeno sobre as folhas e, consequentemente, possam ter evitado o aumento da mortalidade de lagartas com o passar do tempo.

No tratamento com baculovírus (HzSNPV), foi observada mortalidade de $75 \%$ após 8 dias de exposição, o que se deve principalmente a sua forma de atuação na lagarta, que é dependente da infecção de células de epitélio intestinal e consiste em um processo de multiplicação viral que evolui para a morte da lagarta em torno de 7 dias depois da aplicação do inseticida biológico (Bueno et al., 2012). Nesse caso, a chuva também pode ter reduzido a quantidade de propágulo sobre as folhas; no entanto, a aquisição inicial do baculovírus pelas lagartas foi suficiente para o estabelecimento e desenvolvimento da doença, o que proporcionou níveis satisfatórios de controle.

Para $\mathrm{Bt}$ e baculovírus, foi observada mortalidade acumulada, respectivamente, de 90 e $88 \%$ até o final da fase de pupa, com efeitos mais pronunciados de mortalidade no final da fase larval para o $\mathrm{Bt}$ e na fase de pupa para o baculovírus. Apesar de não terem atingido mortalidade total das lagartas, os resultados obtidos com Bt e baculovírus podem ser considerados satisfatórios, pois são inseticidas biológicos que tendem a ser mais sensíveis a fatores ambientais, o que dificulta sua persistência nas plantas. Além disso, a contaminação da lagarta por Bt e baculovírus ocorre exclusivamente pela ingestão, enquanto inseticidas químicos podem atuar também por contato.

Para as lagartas de $2^{-}$instar que receberam folhas colhidas a partir de 24 horas da pulverização, a melhor eficiência de controle foi observada nos tratamentos compostos por clorantraniliprole e flubendiamida (diamidas), com 100\% de mortalidade acumulada aos 3 dias após o início da exposição (Tabela 1). Para o inseticida regulador de crescimento de insetos, metoxifenozida, foram necessários 8 dias para que a mortalidade atingisse $57 \%$; no entanto, a mortalidade acumulada aumentou para $67 \%$ no final da fase larval e foi de $100 \%$ na fase de pupa. Isso indica que, mesmo que não ocorra mortalidade imediata, os efeitos de metoxifenozida podem se manifestar em estádios avançados de desenvolvimento do inseto, como observado por Zarate et al. (2011). Com o inseticida clorfenapir, foi observada mortalidade de $40 \%$ aos 3 dias após a aplicação, tendo chegado a $60 \%$ no final da fase larval e $70 \%$ na fase de pupa. Isso indica que o principal efeito do inseticida ocorreu nos primeiros dias após o início da exposição.

Com o baculovírus foram necessários 8 dias de exposição para que a mortalidade acumulada atingisse $30 \%$, e essa taxa não se alterou até o final da fase larval, mas aumentou drasticamente na fase de pupa, quando foi observada mortalidade de $70 \%$, ou seja, a maior parte dos insetos não conseguiu completar o ciclo.

No tratamento com Bt, não foi observada mortalidade de lagartas até o oitavo dia após o início da exposição, porém, a mortalidade aumentou até o final da fase larval, e na fase de pupa atingiu $60 \%$, o que indica que, nessas condições de exposição, podem ter ocorrido efeitos tardios, e os insetos apresentaram dificuldade para completar o ciclo de desenvolvimento. A transição da fase de lagarta para pupa e desta para adulto implica importantes alterações fisiológicas no inseto, e doses subletais de Bt podem causar mortalidade de pré-pupas e pupas (Burgerjon et al., 1969; Bortoli et al., 2012). Esse efeito também pode ocorrer com plantas Bt, em situação de baixa dose. Tal situação pode acontecer quando lagartas-alvo são expostas a tecidos da planta com baixa expressão da toxina ou quando o tamanho das lagartas lhe proporciona maior tolerância (Kranthi et al., 2005). Esse tema merece investigação detalhada, pois em condição de baixa dose, é maior a probabilidade de desenvolvimento de lagartas resistentes e, nesses casos, é desejável que a mortalidade de pré-pupas e pupas seja de $100 \%$, para evitar que esses indivíduos se reproduzam.

Quando as lagartas de $4^{\circ}$ instar receberam folhas colhidas a partir de 72 horas da pulverização, foi observada mortalidade acumulada de $40 \%$ com 8 dias de exposição, exceto para indoxacarbe, enquanto, nas mesmas condições, para os inseticidas biológicos, essa mortalidade foi de $44 \%$ (Bt) e $11 \%$ (baculovírus) e, em ambos, a mortalidade acumulada evoluiu para 
56 e $33 \%$ na fase de pupa, respectivamente. Para clorantraniliprole e metoxifenozida, a mortalidade evoluiu para $80 \%$ ao final da fase de pupa com efeito mais representativo até o final da fase larval. No caso de indoxacarbe, a mortalidade ocorreu tardiamente principalmente no final da fase larval, tendo chegado a no máximo $50 \%$ na fase de pupa. Isso indica que esse inseticida, na dose avaliada, apresentou baixo efeito residual às 72 horas após a aplicação dos tratamentos. É importante considerar que, em condições de campo, tanto o Bt como o baculovírus podem ser reinoculados e redistribuídos espacialmente no ambiente por meio de propágulos produzidos por lagartas mortas (Praça et al., 2006). Predadores também podem atuar nesse processo, quando se alimentam de lagartas infectadas e excretam o vírus infectivo (Young \& Yearian, 1987), o que pode proporcionar período residual superior ao observado nas condições desse experimento.

Nas avaliações em que as lagartas de $2^{\circ}$ instar foram expostas às folhas coletadas a partir de 72 horas após a pulverização, a eficiência de controle até 8 dias após a exposição não ultrapassou $30 \%$. Os melhores resultados foram observados com clorantraniliprole e flubendiamida (diamidas), com 50 e $60 \%$ de mortalidade acumulada até o final da fase de pupa, respectivamente. Com indoxacarbe, foi observada mortalidade acumulada de $44 \%$ no final da fase larval sem alteração na fase de pupa. Para baculovírus, a mortalidade acumulada até o final da fase de pupa atingiu $50 \%$, com efeito mais importante na fase de pupa. Esses dados indicam reduzido tempo residual para lagartas de 2o instar, o que pode estar relacionado à pequena área foliar do trifólio apical (ponteiro) no momento da pulverização dos tratamentos, o que determina uma pequena quantidade de inseticida depositado inicialmente nessas folhas e prejudica sua disponibilização ao longo do tempo. No entanto, no caso das diamidas, sua capacidade de translocação (Cameron et al., 2015) provavelmente possibilitou um maior aporte de inseticida às folhas apicais, a partir da calda interceptada por outras partes da planta, e contribuiu para um melhor controle de lagartas de $2^{\circ}$ instar a partir de 72 horas da pulverização.

A mortalidade de lagartas que receberam folhas a partir de 72 horas da pulverização foi maior para o $4^{\circ}$ instar do que para o $2^{\circ}$ instar. Isso se deve provavelmente ao fato que as folhas oferecidas para as lagartas de $4^{\circ}$ instar estavam completamente expandidas e encontravam-se no topo do dossel no momento da pulverização, o que possibilitou maior interceptação do inseticida e, consequentemente, permitiu sua ação por mais tempo.

Para biomassa de pupa e deformações da pupa, não houve diferença dos inseticidas entre si e em relação à testemunha, o que indica reduzido efeito dos inseticidas sobre esses parâmetros. As notas de deformação de pupa ficaram próximas a 3,00, o que indicou baixos níveis de deformação (Tabela 1).

Quanto ao tempo letal (TL), para lagartas de 40 instar, expostas a folhas tratadas, a partir de 24 horas da aplicação, os menores TL50 (0,6-0,7 dia) e TL80 (1,1-1,2 dia) foram observados para flubendiamida, clorantraniliprole e clorfenapir. Esses inseticidas apresentam efeito mais rápido, e por isso não diferiram entre si, mas diferiram de todos os demais inseticidas (Tabela 2). Indoxacarbe aparece como um segundo grupo, em ordem crescente, de TL50 e TL80, com valores de 1,7 e 2,4 dias, respectivamente, sendo superior a metoxifenozida em eficiência, com TL50 de 3,2 dias e TL80 de 4,4 dias. Entre os inseticidas biológicos, o Bt apresentou menor TL50 (4,4 dias) em relação ao baculovírus (7,4 dias), o Bt não diferiu de metoxifenozida, enquanto o baculovírus diferiu de todos os tratamentos. Narayanan \& Jayaraj (2002) observam valor de TL50 de 7,0 dias para o baculovírus (HearNPV) oferecido a lagartas de $4^{\circ}$ instar de $H$. armigera. Para lagartas de $3^{\text {o }}$ instar, Gupta et al. (2007) observam TL50 de 5,6-7,3 dias, com variação de acordo com o isolado e a dose do baculovírus. Tanto para Bt como para baculovírus, o TL80 foi superior a 10 dias, sem diferença significativa entre si. Isso indica que esses bioinseticidas apresentam efeito diferenciado em relação aos inseticidas químicos e necessitam de um tempo maior para atingir mortalidade satisfatória. Tal característica precisa ser levada em conta ao utilizar esses produtos biológicos em campo.

Para lagartas de $2^{\circ}$ instar, expostas a folhas tratadas a partir de 24 horas da aplicação, os tratamentos com clorantraniliprole e flubendiamida (diamidas) foram os que apresentaram as menores TL50 e TL80 e diferiram de todos os demais tratamentos (Tabela 2). Os valores de TL80 das diamidas não ultrapassaram 2 dias, o que indicou rápido efeito, pois a maior parte da população de insetos foi controlada com poucos dias após o início da exposição. Metoxifenozida e clorfenapir formam um segundo grupo com TL50 entre 5,5 e 6,2 dias, o 
que representou cerca de quatro vezes mais tempo em relação às diamidas. O TL50 do baculovírus foi de 11 dias, sendo mais rápido do que o $\mathrm{Bt}$ e indoxacarbe. Para todos os inseticidas, exceto as diamidas, o tempo necessário para atingir $80 \%$ de mortalidade (TL80) foi próximo ou superior a 2 semanas.

Quando as lagartas de $4^{\circ}$ instar foram expostas a folhas colhidas a partir de 72 horas da pulverização dos inseticidas, o TL50 variou de 6,9 a 10,1 dias para a maior parte dos inseticidas, exceto indoxacarbe, que apresentou TL50 de 15,8 dias e diferiu de todos os demais inseticidas. O TL80 foi superior a 11 dias em todos os tratamentos. Quando as lagartas de $2^{\circ}$ instar foram expostas a folhas colhidas a partir de 72 horas da pulverização dos inseticidas, o TL50 variou de 10,1 a 15,0 dias, e o TL80 foi superior a 13,3 dias, o que indicou que os inseticidas, nas doses estudadas, apresentaram pequeno efeito residual. Isso mostra que aplicações preventivas ou calendarizadas não protegem satisfatoriamente a lavoura de soja do ataque de $H$. armigera, sendo recomendado realizar o controle da praga apenas quando seu nível de controle for atingido.

O estudo conduzido em campo mostrou controle satisfatório de flubendiamida, espinosade, baculovirus e Bt sobre infestação artificial de lagartas de $2^{\circ}$ e $3^{\circ}$ instar de H. armigera em plantas de soja (Tabela 3). Flubendiamida e espinosade foram os produtos com

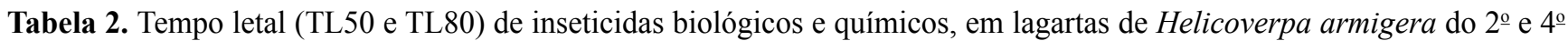
instar, após 24 e 72 horas da pulverização desses produtos em plantas de soja, em Londrina, PR, safra agrícola $2013 / 2014$.

\begin{tabular}{|c|c|c|c|c|c|c|}
\hline \multirow[t]{2}{*}{ Tratamento } & \multicolumn{3}{|c|}{24 horas após a aplicação } & \multicolumn{3}{|c|}{72 horas após a aplicação } \\
\hline & TL $(\operatorname{dias})^{(1)}$ & Limite $(\text { dias })^{(2)}$ & $\mathrm{X}_{\mathrm{c}}^{2(3)}$ & TL $(\operatorname{dias})^{(1)}$ & Limite $(\text { dias })^{(2)}$ & $\mathrm{X}_{\mathrm{c}}^{2(3)}$ \\
\hline & \multicolumn{6}{|c|}{ TL50 - lagartas de $4^{\circ}$ instar } \\
\hline Bacillus thuringiensis & 4,40 & $2,84-5,84$ & 9,80 & 9,00 & $8,00-9,90$ & 9,80 \\
\hline Baculovírus & 7,40 & $6,30-8,30$ & 6,40 & 10,00 & $8,00-11,90$ & 49,30 \\
\hline Clorantraniliprole & 0,70 & $0,08-1,10$ & 4,00 & 10,10 & $7,70-13,50$ & 18,90 \\
\hline Flubendiamida & 0,60 & $0,05-1,08$ & 2,60 & 8,70 & $6,60-11,37$ & 19,40 \\
\hline Metoxifenozida & 3,20 & $2,60-3,80$ & 15,60 & 9,20 & $8,40-10,00$ & 5,00 \\
\hline Clorfenapir & 0,70 & $0,08-1,10$ & 4,00 & 6,90 & $5,50-8,10$ & 15,30 \\
\hline \multirow[t]{2}{*}{ Indoxacarbe } & 1,70 & $1,20-2,10$ & 10,23 & 15,80 & $14,90-16,80$ & 1,50 \\
\hline & \multicolumn{6}{|c|}{ TL80 - lagartas de 4 o instar } \\
\hline Bacillus thuringiensis & 13,39 & $10,00-21,60$ & 9,80 & 12,10 & $11,00-13,60$ & 9,80 \\
\hline Baculovírus & 10,25 & $9,00-12,10$ & 6,40 & 13,53 & $11,40-19,30$ & 49,30 \\
\hline Clorantraniliprole & 1,20 & $0,50-1,90$ & 4,00 & 26,70 & $18,30-62,90$ & 18,90 \\
\hline Flubendiamida & 1,10 & $0,50-1,70$ & 2,60 & 22,45 & $16,00-42,30$ & 19,40 \\
\hline Metoxifenozida & 4,40 & $3,80-5,40$ & 15,60 & 11,50 & $10,60-12,80$ & 5,00 \\
\hline Clorfenapir & 1,20 & $0,40-1,90$ & 4,00 & 12,60 & $10,60-16,40$ & 15,30 \\
\hline \multirow[t]{2}{*}{ Indoxacarbe } & 2,40 & $1,90-3,30$ & 10,20 & 18,30 & $17,10-20,70$ & 1,50 \\
\hline & \multicolumn{6}{|c|}{ TL50 - lagartas de 2o instar } \\
\hline Bacillus thuringiensis & 14,60 & $13,70-15,50$ & 10,40 & 14,70 & $14,10-15,30$ & 5,10 \\
\hline Baculovírus & 11,00 & $9,60-12,30$ & 22,70 & 13,03 & $12,00-14,03$ & 25,00 \\
\hline Clorantraniliprole & 1,30 & $0,80-1,70$ & 4,50 & 11,80 & $9,60-15,00$ & 27,20 \\
\hline Flubendiamida & 1,10 & $0,50-1,50$ & 7,20 & 12,60 & $10,00-17,50$ & 23,20 \\
\hline Metoxifenozida & 5,50 & $3,50-7,40$ & 10,00 & 11,90 & $8,30-19,70$ & 62,00 \\
\hline Clorfenapir & 6,20 & $3,50-8,70$ & 31,90 & 10,10 & $8,90-11,20$ & 18,00 \\
\hline \multirow[t]{2}{*}{ Indoxacarbe } & 20,70 & $17,00-33,00$ & 3,60 & 15,00 & $14,40-15,60$ & 3,00 \\
\hline & \multicolumn{6}{|c|}{ TL80 - lagartas de $2 \stackrel{\circ}{\text { instar }}$} \\
\hline Bacillus thuringiensis & 17,10 & $16,00-19,10$ & 10,40 & 15,90 & $15,30-16,90$ & 5,10 \\
\hline Baculovírus & 15,00 & $13,20-18,40$ & 22,70 & 14,90 & $13,80-16,80$ & 25,00 \\
\hline Clorantraniliprole & 1,90 & $1,50-2,80$ & 4,50 & 21,00 & $16,20-38,80$ & 27,20 \\
\hline Flubendiamida & 1,70 & $1,20-2,60$ & 7,20 & 27,10 & $19,03-65,60$ & 23,20 \\
\hline Metoxifenozida & 13,70 & $10,10-24,00$ & 10,00 & 21,50 & $14,90-112,60$ & 62,00 \\
\hline Clorfenapir & 16,84 & $12,10-32,66$ & 31,90 & 13,30 & $12,00-15,70$ & 18,00 \\
\hline Indoxacarbe & 35,50 & $25,10-91,70$ & 3,60 & 16,00 & $15,40-17,10$ & 3,00 \\
\hline
\end{tabular}

${ }^{(1)}$ Tempo estimado (dias) para serem atingidos 50\% (TL50) e 80\% (TL80) de mortalidade das lagartas. ${ }^{(2)}$ Amplitude do TL (dias). ${ }^{(3)}$ Valor do teste qui-quadrado calculado com 5\% de significância. 
melhor eficiência de controle, com elevada mortalidade (>75\%) já a partir de 3 dias após a pulverização. A eficiência de espinosade no controle de $H$. armigera é amplamente conhecida em culturas como algodoeiro, tomateiro e feijão-guandu (Razaq et al., 2005; Babariya et al., 2010; Ghosh et al., 2010; Chatterjee \& Mondal, 2012). O baculovírus foi mais eficiente para lagartas de $2^{\mathrm{o}}$ instar, tendo atingido $100 \%$ de mortalidade 8 dias após a pulverização, enquanto para as lagartas de 3 instar, a mortalidade atingiu 91,5\%. Os diferentes produtos comerciais que contêm $\mathrm{Bt}$ apresentaram eficiência semelhante entre si, com destaque para as formulações de suspensão concentrada Dipel (Valente BioSciences Corporation, Osage, IA, EUA) e Best HD (Laboratório Farroupilha, Patos de Minas, MG), com mortalidade aos 8 dias após a pulverização que variou de 97,5 a $100 \%$ para lagartas de 2 o instar e de 87,5 a 90,0\% para lagartas de 3 - instar. De modo geral, as lagartas de $2 \circ$ instar foram controladas mais facilmente do que as de $3^{-}$instar, porém, mesmo para estas o controle foi satisfatório.

A maior eficiência de controle por meio de $\mathrm{Bt}$ e baculovírus em campo deveu-se provavelmente à possibilidade de a lagarta ter se alimentado de diferentes partes da planta, não apenas do ponteiro, como ocorreu em laboratório. Ao longo das avaliações realizadas em campo, constatou-se que as lagartas de $2^{\circ}$ instar atacaram outras folhas da planta além do ponteiro. Assim, em campo, houve mais chances de as lagartas de $2 \mathrm{o}$ instar adquirirem os produtos biológicos em relação às avaliadas em laboratório.

Tabela 3. Mortalidade acumulada (\%) de lagartas de Helicoverpa armigera do $2^{\circ}$ e $3^{\circ}$ instar, que infestaram plantas de soja e foram pulverizadas com inseticidas biológicos, espinosade e flubendiamida em campo, em Londrina, PR, safra agrícola 2014/2015 $5^{(1)}$.

\begin{tabular}{lccccc}
\hline Tratamento & \multicolumn{2}{c}{ Lagartas de 2 instar } & & \multicolumn{2}{c}{ Lagartas de 3 instar } \\
\cline { 2 - 3 } \cline { 6 - 6 } & 3 DAP & 8 DAP & & 3 DAP & 8 DAP \\
\hline B. thuringiensis (Dipel WP) & $53,0 \mathrm{c}$ & $90,0 \mathrm{a}$ & & $30,0 \mathrm{~d}$ & $85,0 \mathrm{~b}$ \\
B. thuringiensis (Dipel) & $61,2 \mathrm{c}$ & $97,5 \mathrm{a}$ & & $60,0 \mathrm{c}$ & $90,0 \mathrm{~b}$ \\
B. thuringiensis (Best HD) & $52,5 \mathrm{c}$ & $100,0 \mathrm{a}$ & & $62,5 \mathrm{bc}$ & $87,5 \mathrm{~b}$ \\
Baculovírus (HzSNPV) & $72,5 \mathrm{~b}$ & $100,0 \mathrm{a}$ & & $33,3 \mathrm{~d}$ & $91,5 \mathrm{~b}$ \\
Espinosade & $100,0 \mathrm{a}$ & $100,0 \mathrm{a}$ & & $75,0 \mathrm{~b}$ & $100,0 \mathrm{a}$ \\
Flubendiamida & $77,8 \mathrm{~b}$ & $100,0 \mathrm{a}$ & & $100,0 \mathrm{a}$ & $100,0 \mathrm{a}$ \\
Coeficiente de variação (\%) & 5,7 & 8,0 & 6,7 & 6,7 \\
\hline
\end{tabular}

${ }^{(1)}$ Médias seguidas de letras iguais na coluna não diferem pelo teste de Tukey a $5 \%$ de probabilidade. ${ }^{(2)}$ Mortalidade acumulada, avaliada aos 3 e 8 dias após a pulverização (DAP) dos tratamentos sobre plantas infestadas com lagartas, calculada pela fórmula de Schneider-Orelli (Püntener, 1981).

\section{Conclusões}

1. Os inseticidas flubendiamida, clorantraniliprole, espinosade, clorfenapir, indoxacarbe, metoxifenozida, baculovírus (HzSNPV) e Bt (var. kurstaki HD-1) apresentam desempenho satisfatório para o controle de Helicoverpa armigera em soja.

2. Os inseticidas metoxifenozida, baculovírus (HzSNPV) e Bt (var. kurstaki HD-1) apresentam ação mais lenta que flubendiamida, clorantraniliprole, clorfenapir e indoxacarbe no controle de $H$. armigera.

3. A partir de 72 horas da pulverização, nenhum dos inseticidas testados apresentam controle satisfatório de H. armigera.

\section{Agradecimentos}

À Coordenação de Aperfeiçoamento de Pessoal de Nível Superior (Capes) e ao Programa de Pós-Graduação em Agronomia, Agricultura e Ambiente da Universidade Federal de Santa Maria, Campus de Frederico Westphalen, pela concessão de bolsa; e às equipes de laboratório e campo do Setor de Entomologia da Embrapa Soja pelo suporte na condução dos experimentos.

\section{Referências}

ABBAS, G.; HASSAN, N.; FARHAN, M.; HAQ, I.; KARAR, $\mathrm{H}$. Effect of selected insecticides on Helicoverpa armigera Hubner (Lepidoptera: Noctuidae) on tomato (Lycopersicon esculentum Miller) and their successful management. Advances in Entomology, v.3, p.16-23, 2015. DOI: 10.4236/ae.2015.31003.

ALAVO, T.B.C.; TÉGBÉSSOU, K.J.C.; YAROU, B.B. Potentialities of methoxyfenozide for the integrated management of Helicoverpa armigera (Lepidoptera: Noctuidae) on cotton in Benin, West Africa. Archives of Phytopathology and Plant Protection, v.44, p.813-819, 2011. DOI: 10.1080/03235401003633881.

BABARIYA, P.M.; KABARIA, B.B.; PATEL, V.N.; JOSHI, M.D. Chemical control of gram pod borer, Helicoverpa armigera Hubner infesting pigeonpea. Legume Research, v.33, p.224-226, 2010.

BORTOLI, S.A. de; VACARI, A.M.; MAGALHÃES, G.O. de; DIBELLI, W.; BORTOLI, C.P. de; ALVES, M.P. Subdosagens de Bacillus thuringiensis em Plutella xylostella (Lepidoptera: Plutellidae) e Trichogramma pretiosum (Hymenoptera: Trichogrammatidae). Revista Caatinga, v.25, p.50-57, 2012.

BUENO, A. de F.; SOSA-GÓMEZ, D.R.; CORRÊA-FERREIRA, B.S.; MOSCARDI, F.; BUENO, R.C.O. de F. Inimigos naturais das pragas da soja. In: HOFFMANN-CAMPO, C.B.; CORRÊA-FERREIRA, B.S.; MOSCARDI, F. (Ed.). Soja: manejo integrado de insetos e outros artrópodes-praga. Brasília: Embrapa, 2012. p.493-629. 
BURGERJON, A.; BIACHE, G.; CALS, P. Teratology of the Colorado potato beetle, Leptinotarsa decemlineata, as provoked by larval administration of the thermostable toxin of Bacillus thuringiensis. Journal of Invertebrate Pathology, v.14, p.274-278, 1969. DOI: 10.1016/0022-2011(69)90117-7.

CAMERON, R.A.; WILlIAMS, C.J.; PORTILlO, H.E.; MARÇON, P.C.; TEIXEIRA, L.A. Systemic application of chlorantraniliprole to cabbage transplants for control of foliar-feeding lepidopteran pests. Crop Protection, v.67, p.13-19, 2015. DOI: 10.1016/j.cropro.2014.09.009.

CAMPOS, H. de. Estatística experimental não-paramétrica. 3.ed. Piracicaba: Esalq, 1979. 343p.

CHATTERJEE, M.L.; MONDAL, S. Sustainable management of key lepidopteran insect pests of vegetables. Acta Horticulturae, v.958, p.147-153, 2012. DOI: 10.17660/ActaHortic.2012.958.17.

CUNNINGHAM, J.P.; ZALUCKI, M.P. Understanding heliothine (Lepidoptera: Heliothinae) pests: what is a host plant? Journal of Economic Entomology, v.107, p.881-896, 2014. DOI: 10.1603/ EC14036.

CZEPAK, C.; ALBERNAZ, K.C.; VIVAN, L.M.; GUIMARÃES, H.O.; CARVALHAIS, T. Primeiro registro de ocorrência de Helicoverpa armigera (Hübner) (Lepidoptera: Noctuidae) no Brasil. Pesquisa Agropecuária Tropical, v.43, p.110-113, 2013. DOI: $10.1590 /$ S1983-40632013000100015.

DHADIALLA, T.S. (Ed.). Advances in insect physiology: insect growth disruptors. London: Elsevier, 2012. 552p.

EUROPEAN AND MEDITERRANEAN PLANT PROTECTION ORGANIZATION. Distribution maps of quarantine pests: Helicopverpa armigera. European and Mediterranean Plant Protection Organization, 2006. 6p. Available at: $<$ https://www. eppo.org/QUARANTINE/insects/Helicoverpa_armigera/ HELIAR_map.htm>. Accessed on: 4 Set. 2009.

FARROW, R.A.; DALY, J.C. Long-range movements as an adaptive strategy in the genus Heliothis (Lepidoptera: Noctuidae): a review of its occurrence and detection in 4 pest species. Australian Journal of Zoology, v.35, p.1-24, 1987. DOI: 10.1071/ ZO9870001.

GHOSH, A.; CHATTERJEE, M.; ROY, A. Bio-efficacy of spinosad against tomato fruit borer (Helicoverpa armigera Hub.) (Lepidoptera: Noctuidae) and its natural enemies. Journal of Horticulture and Forestry, v.2, p.108-111, 2010.

GREENE, G.L.; LEPPLA, N.C.; DICKERSON, W.A. Velvetbean caterpillar: a rearing procedure and artificial medium. Journal of Economic Entomology, v.69, p.487-488, 1976. DOI: 10.1093/ jee/69.4.487.

GUPTA, R.K.; RAINA, J.C.; ARORA, R.K.; BALI, K. Selection and field effectiveness of Nucleopolyhedrovirus isolates against Helicoverpa armigera (Hubner). International Journal of Virology, v.3, p.45-59, 2007. DOI: 10.3923/IJV.2007.45.59.

HERMAN, J.C. (Ed.). How a soybean plant develops. Ames: Iowa State University of Science and Technology Cooperative Extension Service, 1985. 20p. (Special Report, 53).

JOHNSON, M.-L.; ZALUCKI, M.P. Foraging behavior of Helicoverpa armigera first instar larvae on crop plants of different developmental stages. Journal of Applied Entomology, v.129, p.239-245, 2005. DOI: 10.1111/j.1439-0418.2005.00958.x.

KARIM, S. Management of Helicoverpa armigera: a review and prospectus for Pakistan. Pakistan Journal of Biological Sciences, v.3, p.1213-1222, 2000. DOI: 10.3923/pjbs.2000.1213.1222.

KRANTHI, K.R.; NAIDU, S.; DHAWAD, C.S.; TATWAWADI, A.; MATE, K.; PATIL, E.; BHAROSE, A.A.; BEHERE, G.T.; WADASKAR, R.M.; KRANTHI, S. Temporal and intra-plant variability of Cry1Ac expression in Bt-cotton and its influence on the survival of the cotton bollworm, Helicoverpa armigera (Hübner) (Noctuidae: Lepidoptera). Current Science, v.89,p.291-298, 2005.

LAMMERS, J. W.; MACLEOD, A. Report of a pest risk analysis: Helicoverpa armigera (Hübner, 1808). 2007. 18p. Available at: $<$ http://webarchive.nationalarchives.gov.uk/20140904082245/ http://www.fera.defra.gov.uk/plants/plantHealth/pestsDiseases/ documents/helicoverpa.pdf $>$. Accessed on: 28 Set. 2015.

MIRONIDIS, G.K.; STAMOPOULOS, D.C.; SAVOPOULOUSOULTANI, M. Overwintering survival and spring emergence of Helicoverpa armigera (Lepidoptera: Noctuidae) in Northern Greece. Environmental Entomology, v.39, p.1068-1084, 2010. DOI: 10.1603/EN09148.

MURÚA, M.G.; SCALORA, F.S.; NAVARRO, F.R.; CAZADO, L.E.; CASMUZ, A.; VILLAGRÁN, M.E.; LOBOS, E.; GASTAMINZA, G. First record of Helicoverpa armigera (Lepidoptera: Noctuidae) in Argentina. Florida Entomologist, v.97, p.854-856, 2014. DOI: 10.1653/024.097.0279.

NARAYANAN, K.; JAYARAJ, S. Mass production of polyhedral occlusion bodies of NPV of Helicoverpa armigera in relation to dose, age and larval weight. Indian Journal of Experimental Biology, v.40, p.846-849, 2002.

NASERI, B.; FATHIPOUR, Y.; MOHARRAMIPOUR, S.; HOSSEININAVEH, V. Comparative life history and fecundity of Helicoverpa armigera (Hubner) (Lepidoptera: Noctuidae) on different soybean varieties. Entomological Science, v.12, p.147-154, 2009a. DOI: 10.1111/j.1479-8298.2009.00310.x.

NASERI, B.; FATHIPOUR, Y.; MOHARRAMIPOUR, S.; HOSSEININAVEH, V. Life Table parameters of the cotton bollworm, Helicoverpa armigera (Lep.: Noctuidae) on different soybean cultivars. Journal of Entomological Society of Iran, v.29, p.25-40, 2009 b.

PEDGLEY, D.E. Windborne migration of Heliothis armigera (Hübner) (Lepidoptera: Noctuidae) to the British Isles. Entomologist's Gazette, v.36, p.15-20, 1985.

PRAÇA, L.B.; SILVA NETO, S.P. da; MONNERAT, R.G. Anticarsia gemmatalis Hübner, 1818 (Lepidoptera: Noctuidae) biologia, amostragem e métodos de controle. Brasília: Embrapa Recursos Genéticos e Biotecnologia, 2006. 18p. (Embrapa Recursos Genéticos e Biotecnologia. Documentos, 196).

PÜNTENER, W. Manual for field trials in plant protection. $2^{\text {nd }}$ ed. rev. and enl. Basle: Ciba-Geigy, 1981.

RAZAQ, M.; SUHAIL, A.; ASLAM, M.; ARIF, M.J.; SALEEM, M.A.; KHAN, M.H.A. Evaluation of new chemistry and conventional insecticides against Helicoverpa armigera (Hubner) 
on cotton at Multan (Pakistan). Pakistan Entomologist, v.27, p.71-73, 2005.

RODRIGUES FILHO, I.L. Comparação de dietas artificiais para Heliothis virescens (Fabr., 1781) (Lepidoptera: Noctuidae) através de estudos biométricos e nutricionais. 1985. 86p. Dissertação (Mestrado) - Escola Superior de Agricultura "Luiz de Queiroz", Universidade de São Paulo, Piracicaba, SP.

SENAVE en alerta tras ingreso de peligrosa plaga agrícola. ABC Color, [Asunción], 17 oct. 2013. Disponível em: <http:// www.abc.com.py/edicion-impresa/economia/ingreso-depeligrosa-plaga-agricola-629240.html>. Acesso em: 1 nov. 2014.

SISLEGIS: Sistema de consulta à legislação. 2014. Disponível em: $<$ http://sistemasweb.agricultura.gov.br/sislegis/>. Acesso em: 16 nov. 2014.

SPECHT, A.; SOSA-GÓMEZ, D.R.; PAULA-MORAES, S.V. de; YANO, S.A.C. Identificação morfológica e molecular de Helicoverpa armigera (Lepidoptera: Noctuidae) e ampliação de seu registro de ocorrência no Brasil. Pesquisa
Agropecuária Brasileira, v.48, p.689-692, 2013. DOI: 10.1590/ S0100-204X2013000600015.

TAY, W.T.; SORIA, M.F.; WALSH, T.; THOMAZONI, D.; SILVIE, P.; BEHERE, G.T.; ANDERSON, C.; DOWNES, S. A brave New World for an Old World pest: Helicoverpa armigera (Lepidoptera: Noctuidae) in Brazil. Plos One, v.8, p.1-7, 2013. DOI: 10.1371/ journal.pone.0080134.

YOUNG, S.Y.; YEARIAN, W.C. Nabis roseipennis adults (Hemiptera: Nabidae) as disseminators of nuclear polyhedrosis virus to Anticarsia gemmatalis (Lepidoptera: Noctuidae) larvae. Environmental Entomology, v.16, p.1330-1333, 1987. DOI: 10.1093/ee/16.6.1330.

ZARATE, N.; DÍAZ, O.; MARTÍNEZ, A.M.; FIGUEROA, J.I.; SCHNEIDER, M.I.; SMAGGHE, G.; VIÑUELA, E.; BUDIA, F.; PINEDA, S. Lethal and sublethal effects of methoxyfenozide on the development, survival and reproduction of the fall armyworm, Spodoptera frugiperda (J. E. Smith) (Lepidoptera: Noctuidae). Neotropical Entomology, v.40, p.129-137, 2011. DOI: 10.1590/ S1519-566X2011000100020.

Recebido em 30 de novembro de 2014 e aprovado em 19 de outubro de 2015 